\title{
Morbidade e mortalidade associadas ao fechamento de colostomias e ileostomias em alça acessadas pelo estoma intestinal
}

\author{
Morbidity and mortality associated to loop colostomy and ileostomy closure
}

Ricardo Augusto Nahuz de Oliveira'; Paulo Gonçalves de Oliveira, TCBC-DF²; Antônio Carlos Nobrega dos Santos, ACBC-DF; JoÃo BATISTA de SOUSA, TCBC-DF²

R E S U M O

\begin{abstract}
Objetivo: Avaliar a morbimortalidade de operações realizadas para fechamento de colostomias e ileostomias em alça. Métodos: Foram analisados os dados epidemiológicos, complicações pós-operatórias, morbidade e mortalidade de pacientes que se submeteram à operações para fechamento de colostomias e ileostomias em alça. Foram excluídos os pacientes cujos dados não puderam ser obtidos nos prontuários e os que operações para fechamento requereram laparotomia mediana. Resultados: Foram operados 88 pacientes, sendo cinco excluídos. Foram avaliados os dados de 83 pacientes, 56 pacientes com colostomias (grupo C) e 27 com ileostomias (grupo I). O sexo masculino predominou em ambos os grupos (grupo C = 71,9\% e grupo I = 57,7\%). No grupo $C$ a indicação mais comum para a confecção do estoma foi trauma abdominal $(43,9 \%)$ e no grupo I foi proteção de anastomose colorretal (57,6\%). A taxa de deiscência de anastomose no grupo C foi 3,5\% e no grupo I foi 19,2 \%. A morbidade no grupo I foi maior do que no grupo C $(30,7 \% \times 12,2 \%)$. Ocorreu um óbito no grupo I. Conclusão: $O$ estudo sugere que taxas de morbidade associadas ao fechamento do estoma são altas e foram maiores no grupo de pacientes com ileostomia em alça.
\end{abstract}

Descritores: Colostomia. Ileostomia. Complicações pós-operatórias. Morbidade. Mortalidade.

\section{INTRODUÇÃO}

\begin{abstract}
\s operações para confecção de estomas em alça são Afrequentes na prática cirúrgica, e são principalmente indicadas para a proteção de anastomoses colorretais baixas e para tratar as lesões ocorridas após trauma abdominal ${ }^{1-7}$. Os estomas determinam diminuição na qualidade de vida dos pacientes e a morbidade associada, em especial aquela relacionada à operação para o seu fechamento, varia entre $10 \%$ e $60 \%$ em alguns estudos ${ }^{1-13}$.

Nesse sentido, alguns autores procuraram identificar fatores determinantes de complicações relacionadas a operações para fechamento de estomas. O sítio do estoma, o tempo de permanência com o estoma, a técnica operatória, o preparo intestinal utilizado antes do fechamento, o uso de antibiótico profilático e o tipo de anastomose são citados em algumas séries ${ }^{1-4,13-16}$.

Esse estudo objetiva avaliar dados epidemiológicos, complicações pós-operatórias, morbidade e mortalidade em dois grupos de pacientes que se submeteram à operação para fechamento de colostomias e ileostomias em alça.
\end{abstract}

\section{MÉTODOS}

Trata-se de um estudo retrospectivo em que foram avaliados os prontuários de pacientes portadores de estomas em alça, que se submeteram à operação para reconstrução do trânsito intestinal entre janeiro de 1991 e dezembro de 2005, no Serviço de Coloproctologia do Hospital Universitário Brasília. Os prontuários dos pacientes foram localizados a partir dos registros de operações realizadas no serviço e foi confeccionado protocolo de avaliação, que levou em consideração os seguintes aspectos: dados epidemiológicos, características da operação, indicação para a confecção do estoma, comorbidades, complicações operatórias e tempo de internação.

Foram consideradas as seguintes complicações cirúrgicas ocorridas até 30 dias após o ato operatório: deiscência de anastomose, infecção de sítio cirúrgico, fístula êntero-cutânea, evisceração, morbidade, mortalidade.

Foram coletados dados relativos ao tipo de preparo mecânico de cólon, antibioticoprofilaxia com os esquemas de antibióticos utilizados, tempo operatório, tipo

Trabalho realizado no Hospital Universitário de Brasília - Serviço de Coloproctologia da Faculdade de Medicina da Universidade de Brasília. Área de Clínica Cirúrgica.

1. Professor Associado de Clínica Cirúrgica da Faculdade de Medicina da Universidade Brasília- Brasília - DF-BR; 2. Professor adjunto de clínica Cirúrgica da Faculdade de Medicina da Universidade Brasília; 3. Cirurgião do Serviço de Coloproctologia do Hospital Universitário de Brasília. 
de anestesia e características da anastomose intestinal realizada, se mecânica ou manual.

Foram excluídos do estudo os pacientes cujos dados não puderam ser obtidos nos prontuários. Também foram excluídos da avaliação os pacientes cujas operações para reconstrução do trânsito intestinal requereram laparotomia mediana. Denominou-se de grupo C e grupo I os pacientes que possuíam colostomia e ileostomia, respectivamente.

As comorbidades foram avaliadas no período préoperatório por anestesiologistas que determinaram o estado físico dos pacientes segundo a classificação da Associação Americana de Anestesiologitas (ASA) ${ }^{17}$.

O teste do $\chi^{2}$ foi utilizado para comparação da classificação ASA e de diagnóstico de câncer. O teste de Fisher foi empregado para avaliar taxa de deiscência, a morbidade e as indicações para realização dos estomas.

\section{RESULTADOS}

Foram operados 88 pacientes e estudados 83 , sendo 57 com colostomias (grupo C) e $26 \mathrm{com}$ ileostomias (grupo I). Cinco pacientes foram excluídos: quatro por falta de dados nos prontuários e, um por ter necessitado de incisão mediana para o fechamento da ileostomia.

Os dados epidemiológicos, as indicações de confecção do estoma, as características das operações de reconstrução do trânsito intestinal, as complicações cirúrgicas, a morbidade, a mortalidade e o tempo de internação são apresentados nas tabelas 1 a 4.

\section{DISCUSSÃO}

A análise dos dados epidemiológicos (Tabela 1) permite observar que a idade e a classificação ASA foram semelhantes entre pacientes dos grupos $\mathrm{C}$ e I, sendo a maioria dos pacientes classificada como ASA II. A mediana da idade foi semelhante em ambos os grupos e comparável a apresentada em outros estudos 1,3,6,8,16.18,19. Em outras séries observou-se a predominância do sexo masculino, especialmente em pacientes vítimas de traumatismo abdominal ${ }^{4-6,18-22}$, esta predominância, no presente estudo, repete-se, em especial, no grupo $C$.

Tabela 1 - Dados epidemiológicos.

\begin{tabular}{|c|c|c|}
\hline & Grupo C & Grupo I \\
\hline Idade em anos (mediana) & $39(16-74)$ & $40,5(17-84)$ \\
\hline \multicolumn{3}{|l|}{ Sexo: } \\
\hline Masculino & $71,9 \% \quad(41)$ & $57,7 \% \quad(15)$ \\
\hline Feminino & $28,1 \% \quad(16)$ & $42,3 \% \quad(11)$ \\
\hline Tempo com estoma ( meses) & $7 \quad(2-108)$ & $4 \quad(2-74)$ \\
\hline \multicolumn{3}{|l|}{ Classificação ASA: * } \\
\hline ASA I & $38,5 \% \quad(22)$ & $30,7 \%$ \\
\hline ASA $\|$ & 49,2 & $57,6 \% \quad(15)$ \\
\hline ASA III & $12,2 \%$ & $11,5 \%$ \\
\hline Diagnóstico de cancer colorretal* & $36,8 \% \quad(21)$ & $50 \%$ \\
\hline
\end{tabular}

* $p>0,05$

Tabela 2 - Dados referentes ao preparo pré-operatório, anestesia e aos procedimentos cirúrgicos.

\begin{tabular}{lcccc}
\hline & \multicolumn{2}{c}{ GRUPO C } & GRUPO I \\
\hline Tempo operatório (mediana) & $110 \min (65-200)$ & $90 \min (50-115)$ \\
Preparo mecânico * & $100 \%$ & $100 \%$ & & \\
Antibiótico profilático (gentamicina e metronidazol) & $100 \%$ & $100 \%$ & & \\
Anestesia: & & & & \\
$\quad$ Bloqueio & $94,7 \%$ & $(54)$ & $36,1 \%$ & $(25)$ \\
$\quad$ Geral & $5,3 \%$ & $(3)$ & $3,9 \%$ & $(1)$ \\
Anastomose: & $92,9 \%$ & $(53)$ & $96,2 \%$ & $(25)$ \\
$\quad$ Manual & $7,1 \%$ & $(4)$ & $3,8 \%$ & $(1)$ \\
$\quad$ Mecânica & &
\end{tabular}

* Solução de manitol, via oral, a 10\% - 2000m/ para fechamento de colostomias e $1000 \mathrm{ml}$ para fechamento de ileostomias. 
Tabela 3 - Evolução pós-operatória.

\begin{tabular}{|c|c|c|c|}
\hline & GRUPO C & GRUPO I & TOTAL \\
\hline $\begin{array}{l}\text { Deiscência de anastomose* } \\
\text { Infecção de sítio cirúrgico }\end{array}$ & $3,5 \%$ & $19,2 \%$ & $8,4 \%$ \\
\hline Superficial & $10,5 \%$ & $3,8 \%$ & $8,4 \% \quad(7)$ \\
\hline Profunda & $1,75 \% \quad(1)$ & $3,8 \%$ & $2,4 \% \quad(2)$ \\
\hline Fístula êntero-cutânea & $1,75 \%(1)$ & $7,6 \%$ & $3,6 \% \quad(3)$ \\
\hline Evisceração & - & $3,8 \%$ & $1,2 \% \quad(1)$ \\
\hline Obstrução intestinal & - & $3,8 \%$ & $1,2 \% \quad(1)$ \\
\hline Morbidade* & $12,25 \%$ & $30,4 \%$ & $18 \% \quad(15)$ \\
\hline Mortalidade & - & $3,8 \%$ & $1,2 \% \quad(1)$ \\
\hline Tempo médio de internação & $6 \operatorname{dias}(4-35)$ & 6,5 dias $(4-22)$ & $6 \operatorname{dias}(4-35)$ \\
\hline
\end{tabular}

${ }^{*} p<0,0-5$

Tabela 4 - Indicações para confecção do estoma.

\begin{tabular}{|c|c|c|c|}
\hline & GRUPO C (57) & GRUPO I (26) & TOTAL (83) \\
\hline Trauma* & $44,6 \%$ & $11,1 \%$ & $33,7 \% \quad(28)$ \\
\hline Deiscência de anastomose & $25 \%$ & $18,5 \%$ & $22,8 \% \quad(19)$ \\
\hline Proteção de anastomose * & $17,8 \% \quad(10)$ & $55,5 \% \quad(15)$ & $30,1 \%(25)$ \\
\hline Outros & $16 \%$ & $3,7 \%$ & $12 \%$ \\
\hline
\end{tabular}

* $p<0.05$

O tempo de permanência com estoma foi menor no grupo I do que no grupo C, e foi maior que o citado em outros estudos ${ }^{1,18}$, para ambos os grupos, porém, foi semelhante ao encontrado em outro estudo realizado no Brasil $^{19}$

A incidência de pacientes com diagnóstico de câncer colorretal foi maior no grupo de pacientes com ileostomias em alça, porém esta diferença não foi estatisticamente significante. No Serviço de Coloproctologia do Hospital Universitário de Brasília, a confecção de ileostomia em alça tem sido utilizada, de rotina, para proteção de anastomoses colorretais baixas ou coloanais, especialmente as confeccionadas após ressecção de tumores de reto distal ou após proctocolectomia restauradora.

Vários estudos procuraram determinar os fatores associados às complicações encontradas após operações para fechamento de estomas. Estes fatores seriam: drenos intra-abdominais, preparo intestinal mecânico, antibioticoprofilaxia, técnica cirúrgica, doença de base e tempo de permanência com estoma ${ }^{1,3,4,12-15}$. Apesar disso, os resultados obtidos nos estudos são conflitantes ao relacionar tais fatores à morbidade associada ao procedimento. A maioria deles, porém, parece concordar com o fato de que operações para fechamento de estomas possuem morbidade semelhante às operações colorretais de grande porte $1,3,4,12-15$.

O tempo operatório, na presente série, foi semelhante ao verificado em outros estudos ${ }^{1,13}$. Todos os pacientes se submeteram ao preparo intestinal mecânico com solução de manitol a 10\% (volume variável de acordo com o tipo de estoma) e antibioticoprofilaxia. A técnica de confecção das anastomoses foi semelhante nos dois grupos, sendo que na maioria dos casos a sutura manual foi utilizada (Tabela 2). Em recente estudo de metanálise, Lustosa et al. observaram que não há diferenças entre anastomoses mecânicas e manuais quando são analisadas as complicações pós-operatórias, exceto a estenose da anastomose, mais frequente após as suturas mecânicas ${ }^{23}$. Nesse estudo não foram observados pacientes com manifestações clínicas de estenoses de anastomoses.

A análise da tabela 4 nos permite verificar uma clara distinção entre os pacientes avaliados. Dentre os pacientes do grupo $\mathrm{C}$, observamos que o trauma abdominal corresponde à principal indicação para confecção do estoma $(43,85 \%)$, enquanto no grupo I, a proteção de anastomoses intestinais predomina $(57,6 \%)$. No total, a principal indicação para a criação do estoma foi o trauma abdominal (33,7\%). A distinção entre os grupos também se evidencia claramente quando observamos haver uma maior incidência de câncer colorretal no grupo I (Tabela 1).

Tilney et al. em estudo de metanálise, observaram que as operações para fechamento de ileostomias apresentaram taxas de infecção de sitio cirúrgico superficial menores e taxas de obstrução intestinal maiores, quando comparadas ao fechamento de colostomias. No entanto, demonstraram que a morbidade total é semelhante entre os dois tipos de estomas no que concerne à reconstrução do trânsito intestinal ${ }^{24}$. No presente estudo, o grupo C apre- 
sentou taxas de infecção de sitio cirúrgico superficial maiores $(10,8 \% \times 3,8 \%)$ e apenas um paciente do grupo I apresentou obstrução intestinal (3,8\%). A taxa de deiscência de anastomose foi maior no grupo I (19,2\% X 3,5\%), assim como a morbidade $(30,7 \% \times 12,2 \%)$. Há que se considerar tais dados com cautela, visto que este estudo apresenta as limitações inerentes a uma análise retrospectiva. Além disso, os grupos não são homogêneos, em especial no que tange às doenças de base dos pacientes e, ainda, o grupo I é relativamente pequeno. As taxas de deiscência de anastomose, morbidade e mortalidade totais são comparáveis àquelas encontradas na literatura 1,3,13,18,19,23.

O estudo sugere que as taxas de deiscência de anastomose e morbidade de operações para fechamento de estomas em alça são altas, representando um significante problema médico. O fechamento de ileostomias em alças apresentou maior taxa de deiscências do que o de colostomias.

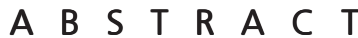

Objective: To evaluate the morbidity and mortality of operations for closure of loop colostomies and ileostomies. Methods: We analyzed epidemiological data, postoperative complications, morbidity and mortality of patients who underwent operations for closure of loop colostomies and ileostomies. We excluded patients whose data could not be obtained from the files and operations that required laparotomy for closure. Results: 88 patients were operated on, five being excluded. We evaluated the data of 83 patients, 56 patients with colostomies (group C) and 27 with ileostomies (group I). Males predominated in both groups (C = 71.9\% and $I=57.7 \%)$. In group $C$ the most common indication for making the stoma was abdominal trauma (43.9\%) and in group I it was protecting a colorectal anastomosis (57.6\%). The rate of anastomotic dehiscence in group C was 3.5\% and in group I $19.2 \%$. Morbidity was higher in group I than in group C (30.7\% vs. $12.2 \%)$. There was one death in group I. Conclusion: The study suggests that morbidity associated with stoma closure is high, being higher in patients with loop ileostomy.

Key words: Colostomy. Ileostomy. Postoperative complications. Morbidity. Mortality.

\section{REFERENCIAS}

1. Khoury DA, Beck DE, Opelka FG, Hicks TC, Timmcke AE, Gathright JB - Colostomy closure. Dis Colon Rectum 1996; 39(6): 605-9.

2. Haagmans MJ, Brinkert W, Bleichrodt RP, van Goor H, Bremers AJ - Short-term outcome of loop ileostomy closure under local anesthesia: Results of a feasibility study. Dis Colon Rectum 2004; 47: 1930-33.

3. Freund HR, Raniel J, Muggia-Sulam M - Factors affecting the morbidity of colostomy closure. Dis Colon Rectum 1981; 24: 71215 .

4. Garber HI, Morris DM, Eisenstat TE, Coker DD, Annous MO Factors influencing the morbidity of colostomy closure. Dis Colon Rectum 1982; 25: 464-70

5. Edwards DP, Leppington-Clarke A, Sexton R, Heald RJ, Moran BJ - Stoma-related complications are more frequent after transverse colostomy than loop ileostomy: a prospective randomized clinical trial. Br J Surg 2001; 88: 360-3.

6. Sousa JB, Sousa ER, Almeida Filho E, Oliveira PG, Machado EL, Ali BAM, Guidoux CHA - Complicações do Fechamento de Colostomia. Rev. Col. Bras. Cir. 1991; 27(5): 240-3.

7. Adesanya AA, Ekanem EE - A ten-year study of penetrating injuries of the colon. Dis Colon Rectum 2004; 47: 2169-77.

8. Ross L, Abild-Nielsen AG, Thomsen BL, Karlsen RV, Boesen EH, Johansen C - Quality of life of Danish colorectal cancer patients with and without a stoma. Support Care Cancer 2007; 15:50513.

9. Nessa J, Fazio VW, Tekkis O, Connor J, Wu J, Bast J et al. - Longterm outcome and quality of life after continent ileostomy. Dis Colon Rectum 2006; 49: 336-44.

10. Baxter NN, Novotny PJ, Jacobson T, Maidl LJ, Sloan J, YoungFadok TM - A stoma quality of life scale. Dis Colon Rectum 2006;49: 205-12.

11. Gooszen AW, Geelkerken RH, Hermans J, Lagaay MB, Gooszen HG - Temporary descompression after colorectal surgery: randomized comparison of loop ileostomy and loop colostomy. $\mathrm{Br}$ J Surg 1998; 85: 76-9.
12. Gastinger I, Marusch F, Steinert R, Wolff S, Koeckerling F, Lippert $\mathrm{H}$ - Protective defunctioning stoma in low anterior resection for rectal carcinoma. Br J Surg 2005; 92 :1137-42.

13. Shellito PC - Complications of abdominal stoma surgery. Dis Colon Rectum 1998; 41:1562-72.

14. Pokorny $H$, Herkner $H$, Jakesz $R$, Herbst $F$ - Predictors for complications after loop stoma closure in patients with rectal cancer. World J Surg 2006; 30:1488-93.

15. Del Pino A, Orsay CP, Nelson RL, Pearl RK, Cintron JR, Abcarian H et al. - Stoma complications. Dis Colon Rectum 1999; 42: 157580.

16. Riesener KP, Winfried L, Höfer M, Kasperk R, Braun JC, Schumpelick $V$ - Morbidity of ileostomy and colostomy closure: impact of surgical technique and perioperative treatment. World J Surg 1997;21:1038.

17. Eagle KA, Brundage BH, Chaitman BR, Ewy GA, Fleisher LA, Hertzer $N R$, et al. Guidelines for perioperative cardiovascular evaluation for noncardiac surgery. Report of the American College of Cardiology / American Heart Association Task Force on Practice Guidelines. Committee on Perioperative Cardiovascular Evaluation for Noncardiac Surgery. Circulation. 1996;93(6):1278-317.

18. Hull TL, Kobe I, Fazio VW - Comparison of hand sewn with stapled loop ileostomy closures. Dis Colon Rectum 1996;39(10): 1086-9.

19. Curi A, Moreira Junior H, Mascarenhas JCS, Moreira JPT, Almeida AC, Azevedo IF et al. - Morbimortalidade associada à reconstrução do trânsito intestinal - análise de 67 casos. Rev bras Coloproct $2002 ; 22(2)$ : 88-97.

20. Von Bahten LC, Nicoluzzi JEL, Silveira F, Nicollelli GM, Kumagai LY, Lima VZ - Morbimortalidade da reconstrução de trânsito intestinal colônica em hospital universitário - análise de 42 casos. Rev bras Coloproct $2006 ; 26(2)$ : 123-7.

21. Thalheimer A, Better M, Kortuem M, Thiede A, Meyer D - Morbidity of temporary loop ileostomy in patients with colorectal câncer. Dis Colon Rectum 2006; 49: p 1011-17.

22. Santos CHM, Bezerra MM, Bezerra FMM, Paraguassú BR. Perfil do paciente Ostomizado e Complicações Relacionadas ao Estoma. Rev Bras Coloproct, 2007;27(1): 16-9 
23. Lustosa SA, Matos D, Atallah AN, Castro AA - Stapled versus handsewn methods for colorectal anastomosis surgery: a systematic review of randomized controlled trials. São Paulo Med J/Rev Paul Med 2002; 120(5): 132-6.

24. Tilney HS, Sains PS, Lovegrove RE, Reese GE, Heriot AG, Tekkis PP - Comparison of outcomes following ileostomy versus colostomy for defunctioning colorectal anastomoses. World J Surg 2007; 31 1142-51.

Recebido em 15/03/2012

Aceito para publicação em 20/05/2012

Conflitos de interesse: nenhum

Fonte de financiamento: nenhuma

\section{Como citar este artigo:}

Sousa JB, Oliveira RAN, Oliveira PG, Santos ACN. Morbidade e mortalidade associadas ao fechamento de colostomias e ileostomias em alça acessadas pelo estoma intestinal. Rev Col Bras Cir. [periódico na Internet] 2012; 39(5). Disponível em URL: http://www.scielo.br/rcbc

\section{Endereço para correspondência:}

João Batista de Sousa

E-mail: sousajb@unb.br 